
\title{
25 Research Soure \\ Utilization of Fecal Occult Blood Testing and Its Impact on Endoscopic Procedures in Hospitalized Patients
}

\author{
Vinod Kumar ( $\nabla$ vinokuma@iu.edu ) \\ Indiana University Health \\ Shobha Shahani \\ Indiana University Health \\ FNU Jaydev \\ Indiana University Health \\ Jaikumar Khatri \\ Indiana University Health \\ Areeba Kara \\ Indiana University Health
}

\section{Research Article}

Keywords: Fecal occult blood testing, colorectal cancer screening, misuse, hospital acquired anemia, stool testing

Posted Date: July 19th, 2021

DOI: https://doi.org/10.21203/rs.3.rs-711548/v1

License: (c) (i) This work is licensed under a Creative Commons Attribution 4.0 International License. Read Full License 


\section{Abstract}

Objective: To gain insights on the impact of fecal occult blood tests, we evaluated inpatients on whom these tests were performed.

Patients and Methods: This single center, retrospective study was conducted at a large, academic, tertiary care center. Between Jan 1, 2016-Dec 31, 2017, inpatients who developed a drop in hemoglobin $\geq 2$ grams/dL and had an FOBT were identified. Further data was extracted on a random selection of half of these patients. Patients were categorized as having an overt GI bleed (symptoms of melena, hematochezia, or hematemesis) or not.

Results: Over the study period 6,310 patients developed a hemoglobin drop of $\geq 2 \mathrm{grams} / \mathrm{dL}$. Of these $817(12.9 \%)$ had an FOBT and we reviewed 407 (49.8\%) randomly selected patients from this group. Those with missing FOBT results $(n=13)$ were excluded, leaving 394 included in the final analysis. The mean age was 62.7 years with 211 females (53.7\%). FOBTs were performed in $34.6 \%$ of patients despite the presence of overt GI bleeding. In patients without overt GI bleeding, the proportion of patients who underwent endoscopic procedures was higher in those with a positive FOBT than those with negative FOBT $(40.4 \%$ vs $13.2 \%, p<.00001)$. There were no differences in rates of endoscopic evaluation in patients with overt GI bleeding based on FOBT results.

Conclusions: FOBTs continue to be utilized in inpatient settings including in those presenting with overt GI bleeding. In the absence of overt GI bleeding, positive results may drive endoscopic evaluation.

\section{Introduction:}

Gastrointestinal (GI) endoscopic procedures are among the most common procedures performed during hospitalizations.[1] Fecal occult blood tests (FOBT) are highly sensitive and have a higher likelihood of false positive testing results in inpatients. [2] While the utility of FOBTs for colorectal cancer screening in ambulatory settings is established, such testing continues to be used in the inpatient setting for the evaluation of anemia and suspected gastrointestinal bleeding. [3, 4] Anemia during hospitalization is common [5] and often triggers an evaluation for GI bleeding including FOBTs. We undertook a descriptive evaluation of patients at our institution, who developed anemia and in whom an FOBT was performed, to gain insights on the utilization of these tests and their impact on management decisions.

\section{Methods:}

This was a single center, retrospective cohort study conducted at a large, Midwestern, academic, tertiary care center. The protocol was reviewed and approved by the Institutional Review Board of Indiana University (IRB-IU). All methods were carried out in accordance with IRB-IU regulation and guidelines. Given the nature of study and IRB requirements, no informed consent was needed from subjects. The informed consent was waived by the Indiana University IRB. 
Patients admitted to medicine services (family medicine or hospitalist) between January 2016 and December 2017 and developed a hemoglobin drop $\geq 2 \mathrm{gm} / \mathrm{dL}$ during the hospitalization were identified. From this group, we further selected patients who had undergone an FOBT. We undertook a detailed review of a random selection of half the patients who had an FOBT performed.

A data collection form was created in Redcap, a data management tool for research.[6] Extracted data included demographics, the presence or absence of overt GI bleeding on admission, nursing documentation of stool description during the hospital stay, results of the FOBT and details of any procedures if performed. A patient was considered to have presented with 'overt GI bleeding' if hematemesis, melena, black or tarry stools, hematochezia or bright red blood per rectum were noted in the admitting documentation. FOBTs at our institution are performed by onsite laboratories using guaiac based Hemoccult by Beckman Coulter Hemoccult Sensa Developer SDs(http://www.beckmancoulter.com/rapids).

Analysis: Data was downloaded from Redcap for analysis. We compared patients who presented with and without overt GI bleed based on the results of the FOBT (positive or negative) and whether an endoscopic procedure was performed. Descriptive analyses were collated. Categorical variables were compared using the chi-square test or the Fisher's Exact test while continuous variables were analyzed utilizing the Student's t-test. A $p$-value $<0.05$ was considered to be statistically significant.

\section{Results:}

Over the study period 6,310 patients were admitted to the family medicine or hospital medicine services and developed a hemoglobin drop of $\geq 2$ grams/dL. Of these, 817 (12.9\%) underwent an FOBT and we reviewed $407(49.8 \%)$ randomly selected patients from this group. FOBT results were missing for 13 patients who were excluded from further analysis. Here we present results for the 394 patients evaluated and included in the final data analysis. (Supplementary Fig. 1)

The mean age of the sample was 62.7 years with a slightly higher number of females ( $n=211,53.7 \%)$ than males $(n=183,46.3 \%)$. The mean length of stay was 12.7 days. More than a third $(n=136,34.6 \%)$ had overt $\mathrm{Gl}$ bleeding symptoms noted in the admission documentation with melena being the most common symptom $(n=78,57.3 \%)$. Concordance between objective stool descriptions noted by bedside nursing and expected stool appearance based on the admitting symptoms was poor- less than half ( $\mathrm{n}=$ $66,48.5 \%)$ of those presenting with overt GI bleeding by history had stool described as melena or hematochezia in nursing notes. (Table 1) 
Table 1

Demographics of patients

\begin{tabular}{|ll|}
\hline Characteristic & N (\%) \\
\hline Total & 394 \\
\hline Mean age (years) & 62.7 range 18-99 \\
\hline Gender & $211(53.7 \%)$ \\
\hline Female & $183(46.3 \%)$ \\
\hline Male & 8.2 \\
\hline Mean lowest recorded hemoglobin (gm/dL)* & 12.7 \\
\hline Mean length of stay (days) & $136(34.6 \%)$ \\
\hline Had overt GI bleed & \\
\hline Presenting symptoms of Gl bleed from admission documentation & $78(57.3 \%)$ \\
\hline Melena & $40(29.4 \%)$ \\
\hline Hematochezia & $32(23.5 \%)$ \\
\hline Hematemesis & $12(8.8 \%)$ \\
\hline Coffee ground emesis & \\
\hline Objective description of stool by nursing documentation & $150(38 \%)$ \\
\hline No description & $66(48.5 \%)$ \\
\hline Melena or hematochezia in those presenting with overt GI bleed & $23(16.9 \%)$ \\
\hline Normal stools in those presenting with overt Gl bleed & \\
\hline *for those undergoing endoscopy lowest pre procedural hemoglobin & \\
\hline Gl = gastrointestinal & \\
\hline
\end{tabular}

FOBT results were positive in 207 (52.5\%) patients with higher positivity rates in those presenting with overt GI bleeding compared to those without overt GI bleeding symptoms $(79.4 \%$ vs $38.5 \%, p<.00001)$. The proportion of those who underwent endoscopic evaluation was higher in those who presented with symptoms of overt GI bleeding than those who did not ( $83 \%$ vs $23.6 \%, p<.00001)$.

In patients who did not present with overt GI bleeding symptoms, the proportion of patients who underwent endoscopic procedures was higher in those with a positive FOBT than those whose FOBT was negative $(40.4 \%$ vs $13.2 \%, p<.00001)$ The diagnostic yield of the endoscopic procedure was also higher in patients without overt GI bleeding and positive FOBT testing vs negative FOBT testing ( $65 \%$ vs $33 \% p$ 
$=.02$ ). Conversely, in patients presenting with symptoms of overt GI bleeding, no differences in rates of endoscopic evaluation or diagnostic yields were noted based on FOBT results. (Table 2)

Table 2

Outcomes based on FOBT testing result

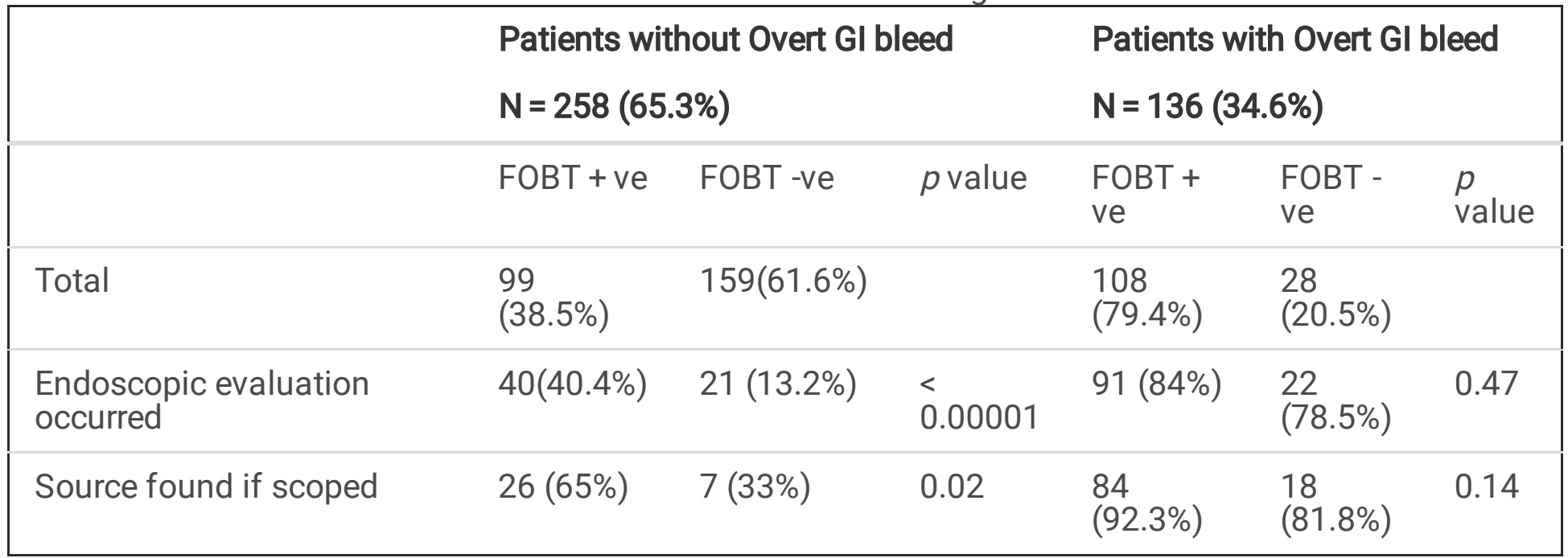

Among the 174 patients who underwent endoscopic evaluation, the most common procedure performed was upper $\mathrm{Gl}$ endoscopy $(\mathrm{n}=150)$. In patients with or without overt $\mathrm{GI}$ bleeding on presentation, the most common pathologies found were gastric ulcers, duodenal ulcers and gastritis. (Table 3) 
Table 3

Description of sources found on endoscopy

\begin{tabular}{|lll|}
\hline & Without overt GI bleed $(\mathbf{n}=\mathbf{3 3})$ & With overt GI bleed $(\mathbf{n}=\mathbf{1 0 2})$ \\
\hline Gastric Ulcer & $10(30.3 \%)$ & $32(31.4 \%)$ \\
\hline Duodenal Ulcer & $10(30.3 \%)$ & $18(17.6 \%)$ \\
\hline Gastritis & $8(24.2 \%)$ & $19(18.6 \%)$ \\
\hline Esophagitis & $6(18.2 \%)$ & $12(11.7 \%)$ \\
\hline Hemorrhoids & $6(18.2 \%)$ & $11(10.7 \%)$ \\
\hline Diverticular bleeding & $2(6 \%)$ & $11(10.7 \%)$ \\
\hline Others & $2(6 \%)$ & $16(15.6 \%)$ \\
\hline Polyps & $2(6 \%)$ & $1(0.9 \%)$ \\
\hline LGI-Malignancy & $2(6 \%)$ & 0 \\
\hline AVM-UGI & $1(3 \%)$ & $7(6.8 \%)$ \\
\hline AVM-LGI & $1(3 \%)$ & 0 \\
\hline Esophageal varices & 0 & $9(8.8 \%)$ \\
\hline Gastric Varices & 0 & $3(2.9 \%)$ \\
\hline UGI-Malignancy & 0 & $2(1.9 \%)$ \\
\hline Abbreviations LGI=Lower Gastro-Intestinal, AVM= Arteriovenous Malformation, UGI= Upper Gastro- & \\
\hline Intestinal & & \\
\hline
\end{tabular}

In comparing patients based on their endoscopic evaluation status, the mean hemoglobin was noted to be lower among those who underwent endoscopic evaluation, whether they presented with symptoms of overt GI bleeding or not. In those presenting with overt GI bleeding, the mean length of stay was shorter among those who underwent endoscopic evaluation. (Table 4) 
Table 4

Characteristics of patients based on endoscopy status

\begin{tabular}{|lllllll|}
\hline & \multicolumn{2}{l}{$\begin{array}{l}\text { Patients without overt GI bleed } \\
\text { N=257 (65.3\%) }\end{array}$} & \multicolumn{3}{l|}{$\begin{array}{l}\text { Patients with overt GI bleed } \\
\mathbf{N = 1 3 6} \text { (34.6\%) }\end{array}$} \\
\hline & Scoped & $\begin{array}{l}\text { Not } \\
\text { scoped }\end{array}$ & $\begin{array}{l}p \\
\text { value }\end{array}$ & Scoped & $\begin{array}{l}\text { Not } \\
\text { scoped }\end{array}$ & $\begin{array}{l}p \\
\text { value }\end{array}$ \\
\hline Total & $\begin{array}{l}61 \\
(23.7 \%)\end{array}$ & $\begin{array}{l}196 \\
(76.3 \%)\end{array}$ & & $\begin{array}{l}113 \\
(83 \%)\end{array}$ & $\begin{array}{l}23 \\
(16.9 \%)\end{array}$ \\
\hline Mean age & 64.2 & 63 & 0.49 & 61.8 & 59.9 & 0.3 \\
$\begin{array}{l}\text { Mean Lowest hemoglobin } \\
\text { (gm/dL) }\end{array}$ & 7.2 & 8.8 & 0.02 & 7.6 & 8.6 & 0.02 \\
\hline Mean length of stay (days) & 15.5 & 14.2 & 0.24 & 8.1 & 12.5 & 0.04 \\
\hline
\end{tabular}

\section{Discussion:}

The current guidelines by the American Gastroenterology Association suggest that fecal occult blood tests should only be used to screen for colorectal cancer (CRC) in average risk, asymptomatic outpatients. [7, 8] [9] As a non-invasive, easily administered and relatively inexpensive test, the FOBT has become a frequently used screening modality for CRC screening across the world.[10] [11, 12] However, the widespread availability of FOBTs has also resulted in their use beyond their sole validated purpose for CRC screening. $[13,14]$. Despite little or no evidence to support the utility of FOBTs in hospitalized patients, studies demonstrate continued inappropriate use in inpatient settings .[15] [16].[17] Notably, FOBTs are frequently used in the evaluation of anemia in hospital settings and we focused our investigation in this population. $[3,17,18]$ While differences in inclusion criteria make it difficult to assess the prevalence of this practice, in our sample, $12.9 \%(n=817)$ of patients who developed a drop in hemoglobin $>2 \mathrm{~g} / \mathrm{dl}$ underwent an FOBT. Most patients $(65.3 \%)$ did not have overt GI bleeding. Concomitant use of certain medications (e.g., acetylsalicylic acid, non-steroidal anti-inflammatory agents, Vitamin C, selective serotonin reuptake inhibitors) and certain foods (e.g., red meat, broccoli, turnips and radishes) can interfere with FOBTs and result in false positive results.[19, 20] Both anemia and the invalidators of FOBTs are common during hospitalization.[4] Given the potential consequences of exposing patients to the risk of unnecessary endoscopic procedures, clinicians should carefully consider the multiple causes of hospital acquired anemia, including blood draws, medications, and bone marrow suppression secondary to acute infections and illness before reflexive ordering of an FOBT.[21, 22]

The use of FOBTs in the setting of overt GI bleeding is also problematic. Rationally, the presence of overt blood should preclude the need for testing for occult blood. Rates of FOBTs performed in the context of overt GI bleed range from $5 \%-23 \%$.[3, 23] In our sample, $34.6 \%$ of those who had FOBTs performed in the setting of a drop in hemoglobin had a history consistent with GI bleed. We also noted a mismatch between the documentation of symptoms consistent with a GI bleed and nursing documentation of stool 
appearance although this may be limited by the absence of documentation in more than half the cases evaluated. Limitations in stool documentation in addition to possible under-utilization of digital rectal examinations to confirm histories may drive inappropriate FOBTs. $(18,19)$ Understanding these drivers of potentially inappropriate testing may help us improve processes and practices.

Previous reports have described variable impacts of FOBT results on subsequent management including delays in care while awaiting testing results, no changes in management and increases in endoscopic procedures. $[3,4,18,23]$ We evaluated patients with and without symptoms of overt GI bleeding separately and found that FOBT results were associated with subsequent endoscopic procedures only in those without overt GI bleeding - where those with a positive FOBT result were more likely to undergo endoscopic evaluation. No association was noted between FOBT results and endoscopy rates in patients with overt GI bleeding. Clinicians may appropriately disregard negative test results when the history is strongly suggestive of a GI bleed but when faced with diagnostic uncertainty, positive FOBT results may lead to confirmation bias and subsequent endoscopic evaluation. We also noted that those with lower hemoglobin values were more likely to have undergone endoscopy regardless of symptoms of GI bleeding. It is likely that clinicians weigh the degree of anemia heavily in their decision to pursue diagnostic endoscopic evaluation.[24] Further study of the interplay between FOBT results, the degree of anemia, subsequent endoscopies and outcomes will help clinicians understand their own cognitive processes and decrease the risk of potentially avoidable procedures.

Interestingly, among patients without overt GI bleeding, we found a higher diagnostic yield from endoscopic procedures with positive FOBT results compared to those with negative FOBTs. The most common pathology found in patients without overt GI bleeding were gastric and duodenal ulcers. It is possible that these lesions bleed intermittently without reaching the threshold of overt GI bleeding. [18] These findings raise the question of whether there are selected clinical scenarios in which FOBTs in the inpatient setting may be helpful.

Our work has certain limitations. It is a single center's experience, and our findings may not be generalizable. We assigned patients to the category of overt GI bleed based on the symptoms documented rather than findings on a digital rectal exam. We started with patients who developed a drop in hemoglobin and therefore cannot comment on inpatient FOBT use driven by other causes. Since this is a retrospective observational study, involving smaller number of patients, we could not do cost-effective analysis.

FOBTs continue to be utilized in inpatient settings for the evaluation of anemia and in those with overt GI bleeding. Understanding the drivers of FOBTs in the inpatient setting, including limitations in stool documentation and possible under-utilization of digital rectal examinations to confirm histories may help us improve processes and practices. Clinicians may appropriately disregard negative test results when the history is strongly suggestive of a Gl bleed but when faced with diagnostic uncertainty, positive results may lead to subsequent potentially unnecessary endoscopic evaluation. 


\section{Declarations:}

\section{Authors Contribution:}

VK: Primary author, Project conceptualization, Data Curation, Data analysis and interpretation, Writingoriginal draft, Writing-review and editing

SS: Data curation, Data analysis, Writing-review and editing

FJ: Data curation, Data analysis, Writing-review and editing

JK: Data analysis, Writing-review and editing

AK: Supervision, Data analysis, writing critical review and editing

\section{Declaration of interests:}

All authors declare that they have no known competing financial interests or personal relationships that could have appeared to influence the work reported in this paper.

\section{Funding:}

None

\section{References:}

1. in Healthcare Cost and Utilization Project (HCUP) Statistical Briefs. 2006, Agency for Healthcare Research and Quality (US): Rockville (MD).

2. Rockey, D.C., et al., Relative frequency of upper gastrointestinal and colonic lesions in patients with positive fecal occult-blood tests. N Engl J Med, 1998. 339(3): p. 153-9.

3. Friedman, A., et al., Use and abuse of faecal occult blood tests in an acute hospital inpatient setting. Intern Med J, 2010. 40(2): p. 107-11.

4. Soin, S., et al., Use and abuse of fecal occult blood tests: a community hospital experience. BMC Gastroenterol, 2019. 19(1): p. 161.

5. Koch, C.G., et al., Hospital-acquired anemia: Prevalence, outcomes, and healthcare implications. Journal of Hospital Medicine, 2013. 8(9): p. 506-512.

6. Harris, P.A., et al., Research electronic data capture (REDCap)--a metadata-driven methodology and workflow process for providing translational research informatics support. J Biomed Inform, 2009. 42(2): p. 377-81.

7. Li, J.N. and S.Y. Yuan, Fecal occult blood test in colorectal cancer screening. J Dig Dis, 2019. 20(2): p. 62-64. 
8. Raju, G.S., et al., American Gastroenterological Association (AGA) Institute technical review on obscure gastrointestinal bleeding. Gastroenterology, 2007. 133(5): p. 1697-717.

9. Levin, B., et al., Screening and surveillance for the early detection of colorectal cancer and adenomatous polyps, 2008: a joint guideline from the American Cancer Society, the US Multi-Society Task Force on Colorectal Cancer, and the American College of Radiology. CA Cancer J Clin, 2008. 58(3): p. 130-60.

10. Rex, D.K., et al., Colorectal Cancer Screening: Recommendations for Physicians and Patients from the U.S. Multi-Society Task Force on Colorectal Cancer. Am J Gastroenterol, 2017. 112(7): p. 1016-1030.

11. Leddin, D., et al., Canadian Association of Gastroenterology and the Canadian Digestive Health Foundation: Guidelines on colon cancer screening. Can J Gastroenterol, 2004. 18(2): p. 93-9.

12. Sharma, V.K., et al., Survey of internal medicine residents' use of the fecal occult blood test and their understanding of colorectal cancer screening and surveillance. Am J Gastroenterol, 2000. 95(8): p. 2068-73.

13. Powell, A.A., et al., Rates and correlates of potentially inappropriate colorectal cancer screening in the Veterans Health Administration. J Gen Intern Med, 2015. 30(6): p. 732-41.

14. Scholefield, J.H., et al., Nottingham trial of faecal occult blood testing for colorectal cancer: a 20-year follow-up. Gut, 2012. 61(7): p. 1036-40.

15. Cuthbert, J.A. and I.A. Hashim, Diagnostic Fecal Occult Blood Testing in Hospitalized and Emergency Department Patients: Time for Change? Laboratory Medicine, 2018. 49(4): p. 385-392.

16. Nadel, M.R., et al., Fecal occult blood testing beliefs and practices of U.S. primary care physicians: serious deviations from evidence-based recommendations. J Gen Intern Med, 2010. 25(8): p. 833-9.

17. Lee, M.W., J.S. Pourmorady, and L. Laine, Use of Fecal Occult Blood Testing as a Diagnostic Tool for Clinical Indications: A Systematic Review and Meta-Analysis. Am J Gastroenterol, 2020. 115(5): p. 662-670.

18. Narula, N., et al., Fecal occult blood testing as a diagnostic test in symptomatic patients is not useful: a retrospective chart review. Can J Gastroenterol Hepatol, 2014. 28(8): p. 421-6.

19. Jaffe, R.M., et al., False-negative stool occult blood tests caused by ingestion of ascorbic acid (vitamin C). Ann Intern Med, 1975. 83(6): p. 824-6.

20. Feinberg, E.J., et al., How long to abstain from eating red meat before fecal occult blood tests. Ann Intern Med, 1990. 113(5): p. 403-4.

21. van Rijn, A.F., et al., Inappropriate use of the faecal occult blood test in a university hospital in the Netherlands. Eur J Gastroenterol Hepatol, 2012. 24(11): p. 1266-9.

22. Ip, S., et al., Use of fecal occult blood test in hospitalized patients: survey of physicians practicing in a large central Canadian health region and Canadian gastroenterologists. Can J Gastroenterol, 2013. 27(12): p. 711-6.

23. Mosadeghi, S., et al., Utilization of fecal occult blood test in the acute hospital setting and its impact on clinical management and outcomes. J Postgrad Med, 2016. 62(2): p. 91-5. 
24. Ioannou, G.N., J. Spector, and D.C. Rockey, Predictors of endoscopic and laboratory evaluation of iron deficiency anemia in hospitalized patients. South Med J, 2007. 100(10): p. 976-84.

\section{Supplementary Files}

This is a list of supplementary files associated with this preprint. Click to download.

- supplementaryfigure.docx 